\title{
The Influence of Rotational Speed on the Wall Thickness and Mechanical Characteristic of Rotomouldable Plastics
}

\author{
Nadhim M. Faleh", Fatima Masood Hani \\ Al-Mustansiriah University, Engineering Collage \\ P.O. Box 46049, Bab-Muadum, Baghdad 10001, IRAQ \\ *Corresponding author's email: drnadhim [AT] gmail.com
}

\begin{abstract}
This study is interested in the main parameter of this technique that uses a cubic container with an internal dimension of $100 \mathrm{~mm}$ and a cylindrical container $100 \mathrm{~mm}$ in diameter. We implemented the work in the fifth stage: In the first stage, we designed and manufactured the multi-axial system. In the second stage, the specimens were moulded from polyester, PVC, and polyethylene at a rotational speed axis of 5-120 rpm. The results from this stage indicated that the optimal rotational speed of the steady quality in dimensions and properties of the parts are 85, 100 and 115 rpm. The third stage was concerned with the effect of the speed of rotation on the thickness of the wall. The rotational speed of the axes was changed, and the thickness of the moulded walls was measured. The laboratory measurements revealed that the maximum compression ratio with the change of speed is at the speed of $115 \mathrm{rpm}$. The fourth stage was concerned with the effect of the speed of rotation on the value of tensile strength. The rotational speed of the axes was changed, and the tensile strength of the mould was measured. The tests revealed an improvement in the tensile strength at the speed of 115 rpm compared with the other speeds. The fifth stage utilised a cylindrical mould, which was re-worked in the previous stages, to investigate the effect of the speed of rotation on the thickness of the wall and mechanical specifications. Based on the conducted experimental tests, the influence of the rotational speed, which characterized the moulding process, on selected geometrical features of the mould was studied and analysed theoretically and numerically. The results showed an increase of about $5 \%$ in the compression ratio with increased rotation speed within the range of 85 to $115 \mathrm{rpm}$. There was also an improvement of about $7 \%$ in the tensile strength with increased rotational speed from 85 to $115 \mathrm{rpm}$. These results are due to the increase in the centrifugal force on the wall of the mould during the process. Also, the study was characterized by the production of the composite of polyethylene reinforced by iron screen wire, with improvement in the mechanical properties by about $300 \%$ compared to the base material.
\end{abstract}

Keywords--- rotational speed, wall thickness, roto-mouldable, plastic

\section{INTRODUCTION}

The main objectives of the study: First; this study is the investigation of the effect of rotational speed on the wall thickness and mechanical properties of mould . Second, An academic attempt to develop the mechanical properties of the mould by using the composite material principle. Third, find theoretical and laboratory knowledge (know how) in the subject of rotational moulding to serving the local needs. the studies and research in this area are few or missed, despite the importance of the subject industrial and developmental.

\section{METHODOLOGY}

In order to achieve the goals and the objectives of the study, we followed these four stages: The first stage included designing and manufacturing of the multi-axial system, using a cubic container with an internal dimension of $100 \mathrm{~mm}$ and a cylindrical container having a diameter of $100 \mathrm{~mm}$. 2. In the second stage, specimens were moulded out of polyester, PVC and polyethylene with a rotational speed axis of 5 $\neg 120 \mathrm{rpm}$. The results of this stage estimated that the optimal rotational speed of the steady quality in dimensions and properties of the parts are 85,100 and $115 \mathrm{rpm}$. 3 . In the third stage, the effect of the speed of rotation on the thickness of the wall was observed. The rotational speed of axes was changed, and the thickness of the mould walls was measured. The laboratory measurements revealed that the maximum compression ratio with the change of speed was $115 \mathrm{rpm}$. 4. The fourth stage was concerned with the effect of the speed of rotation on the value of tensile strength. The rotational speed of axes was changed, and the tensile strength of the mould was measured. The tests revealed an improvement in the tensile strength at a 100rpm speed when compared with the other speeds. 5-The fifth stage included the re-work in the previous stages for a cylindrical mould to investigate the effect of the speed of rotation on the thickness of the wall and mechanical specifications. Based on the conducted experimental tests, the influence of the rotational speed, which characterized the moulding process, on selected geometrical features of the mould has been studied and analysed theoretically and numerically. The sixth stage included moulding specimens of a composite of polyethylene reinforced by iron wire, to improve the mechanical properties compared to the base material. 
The rotational moulding process is a rotation plastic in a liquid or powder resin on two axes. The polymer flows into the mould in the internal. The polymer flowing along the internal walls of the mould is basically determined by centrifugal force. For the multi-rotation axes, the path of a point going to be complex. The process, however, has no correlation between the two axial rotation, physically. Therefore, the rotation can be studied separately for each axis separately. Figures 1 show the description of the process during casting, for both types of moulds, used in this study. The first type is cylindrical container, and the second type is as cube container in shape, respectively.

From figure (1), Centrifugal force+ Gravitational force= viscosity force

$\mathrm{mv}^{\wedge} 2 / \mathrm{r}+\mathrm{mg}=\tau \mathrm{A}$.

$\rho \mathrm{dV} g=\rho \mathrm{dV} \omega^{\wedge} \mathrm{r}=\tau \mathrm{dA}$.

$\omega=\mathrm{v} / \mathrm{r}=3.14 \mathrm{D} \mathrm{N} / 60 \mathrm{r}$

$\rho \mathrm{dV} g=\rho \mathrm{dV}(3.14 \mathrm{D} \mathrm{N} / 60 \mathrm{r})^{\wedge}=\tau \mathrm{dA}$.

Where, m: mass of the body $(\mathrm{kg}), \mathrm{g}$ : Acceleration due to gravity $\left(\mathrm{m} / \mathrm{s}^{\wedge} 2\right), \mathrm{r}$ : Radius of rotation $(\mathrm{m})$

$\mathrm{v}=$ the speed $(\mathrm{m} / \mathrm{s}), \tau=$ the shearing stress between two planes parallel with the direction of flow

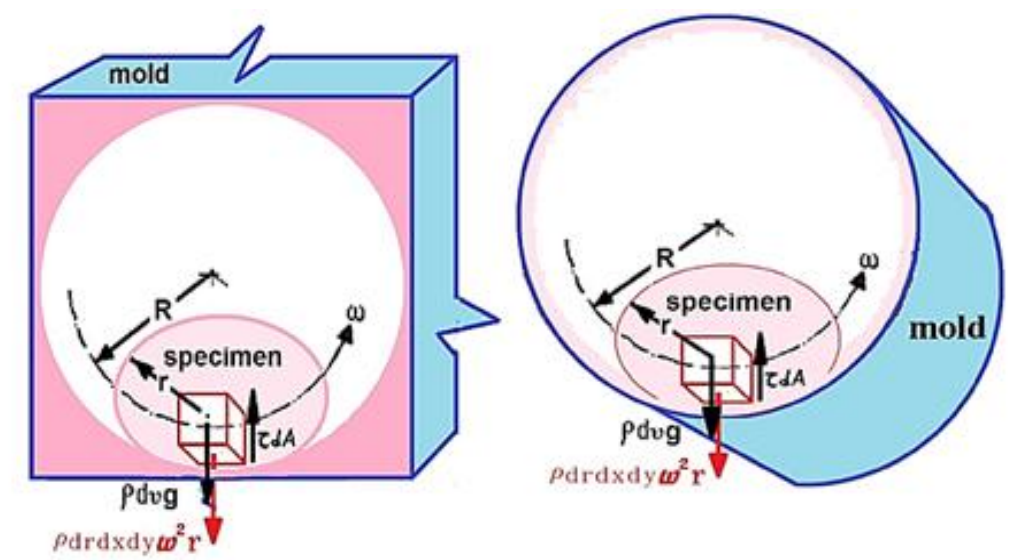

Figure1: The theoretical representation of moulding operation.

Due to Fig. 2, the angle $\theta$ at the semi axes Oz became $(\theta+\omega z t)$, the angle $\beta$ at the semi axes Oy became $(\beta+\omega y t)$ could be considered, the equations system becomes:

$$
\begin{aligned}
\mathrm{X}_{\mathrm{A}} & =R * \sin \left(\tan ^{-1}\left(\frac{1}{\cos (\theta+\omega \mathrm{z} t) * \tan (\beta+\omega \mathrm{yt})}\right)\right) * \cos (\theta+\omega \mathrm{zt}) \\
\mathrm{Y}_{\mathrm{A}} & =R * \sin \left(\tan ^{-1}\left(\frac{1}{\cos (\theta+\omega \mathrm{z}) * \tan (\beta+\omega \mathrm{yt})}\right)\right) * \sin (\theta+\omega \mathrm{zt}) \\
\mathrm{Z}_{\mathrm{A}} & =\mathrm{R}^{*} \cdot \cos \left(\tan ^{-1}\left(\frac{1}{\cos (\theta+\omega \mathrm{zt}) * \tan (\beta+\omega \mathrm{yt})}\right)\right)
\end{aligned}
$$




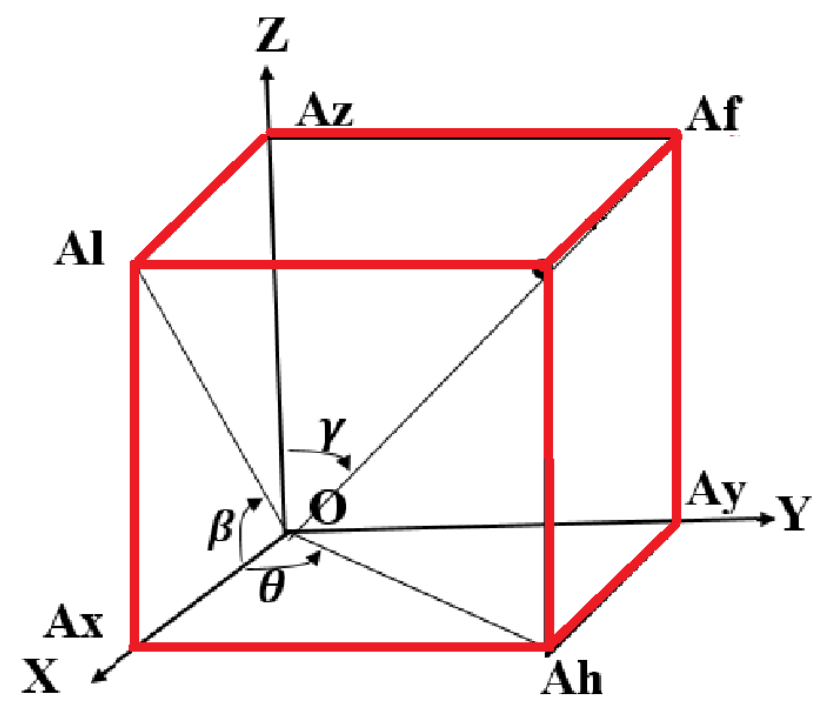

Figure 2: the element of the mold.

\section{EXPERIMENTAL DETAILS}

In order to achieve the objectives of the study, collected information about the rotational moulding system, then we planed the design process. then Hand sketches were developed, then it was converted into digital designs, through available and modern design software. In the first step, the parts are designed, then assembled as the final system. Design using software, provide the study with the opportunity to ensure the possibility of movement and rotation, and the relationship between the parts. The figures illustrate the design processes. The figures 3 illustrate the moulding system.

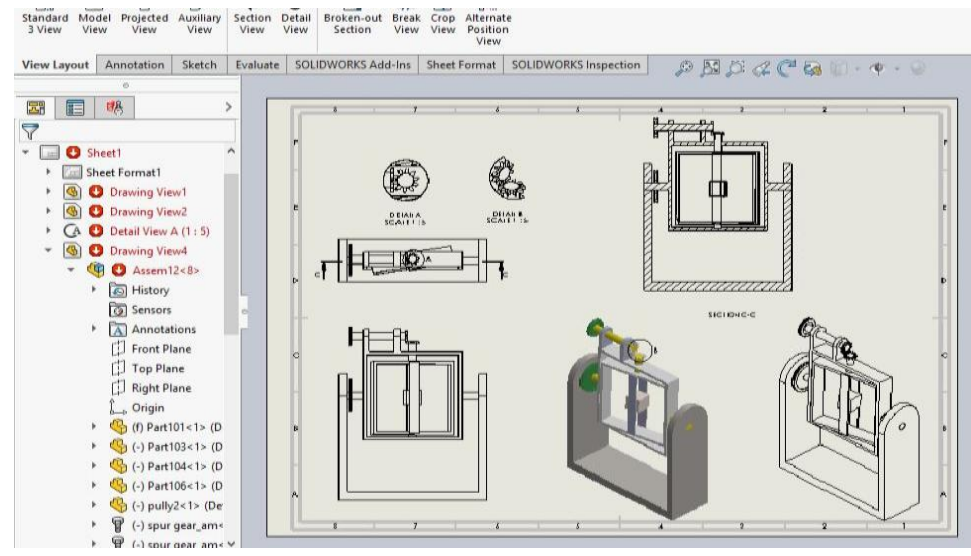

Figure 3: The design of moulding system.

In order to achieve the objectives of the study, information was collected regarding the necessary manufacturing processes, and then planning the manufacturing process. Quantities of materials needed to manufacture the system were calculated. This effectively included cutting and welding. The motor selection was based on the actual weight calculations of the mould and moulded specimen. As well as the selection of power transmission requirements, taking into account the requirements of durability, and the foundations of industrial safety. Table 1 shows the mechanical properties of polymer. Figure 4 and 5 show the roto-mould system. Figure 6 and 7 show the roto-mould specimens. 
Table 1: The mechanical properties of polymer

\begin{tabular}{|c|c|c|c|c|c|}
\hline Polymer & Density & $\begin{array}{c}\text { Tensile } \\
\text { Strength } \\
(\mathrm{MPa})\end{array}$ & $\begin{array}{c}\text { Young's } \\
\text { Modulus } \\
\mathrm{E}(\mathrm{GPa})\end{array}$ & Elongation (\%) & Poison Ratio \\
\hline Epoxy & 1.25 & 0.04 & $3.5-17$ & $10-1$ & 0.38 \\
\hline Polyester & 1.37 & 48 & 2 & $300-5$ & 0.38 \\
\hline Polyethylene & $0.94-0.97$ & $20-38$ & $0.1-1.4$ & $1000-15$ & - \\
\hline PVC & 1.39 & $7-55$ & $0.014-4$ & $20-40$ & 0.46 \\
\hline
\end{tabular}

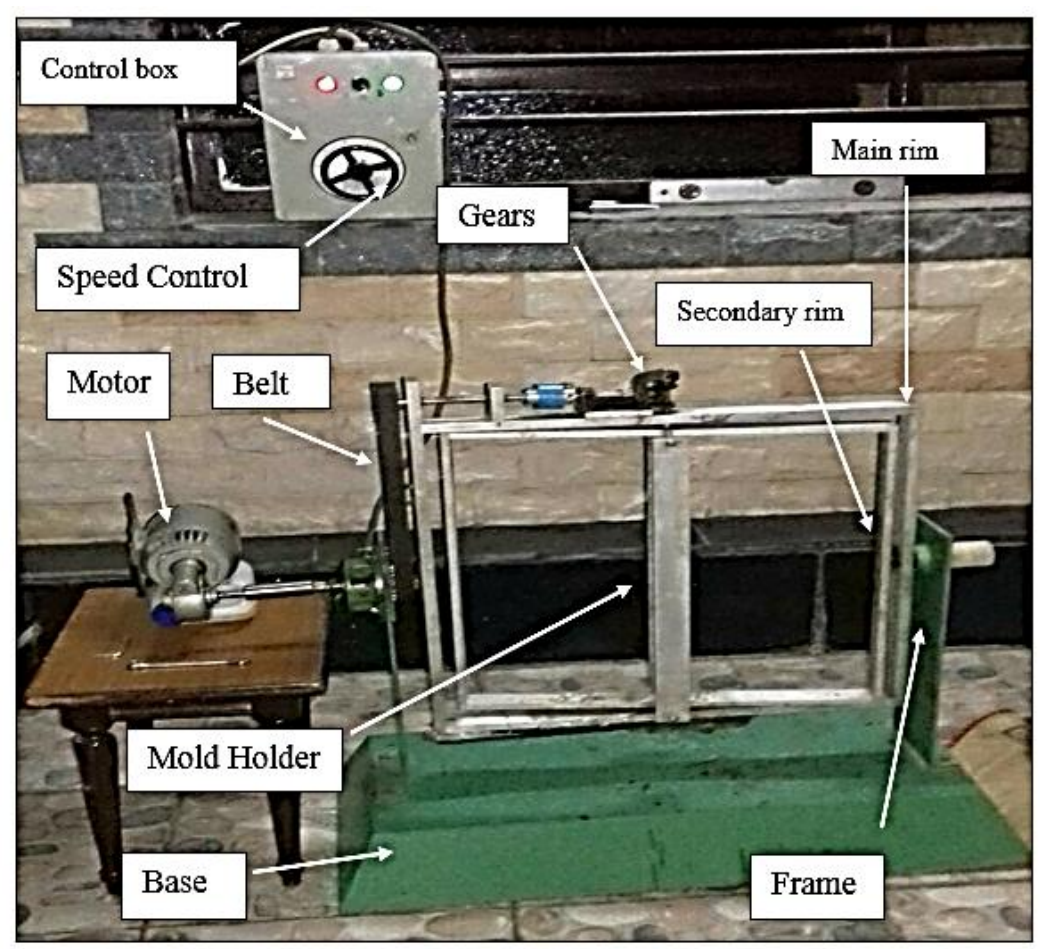

Figure 4: Multi-axis roto-mould system.

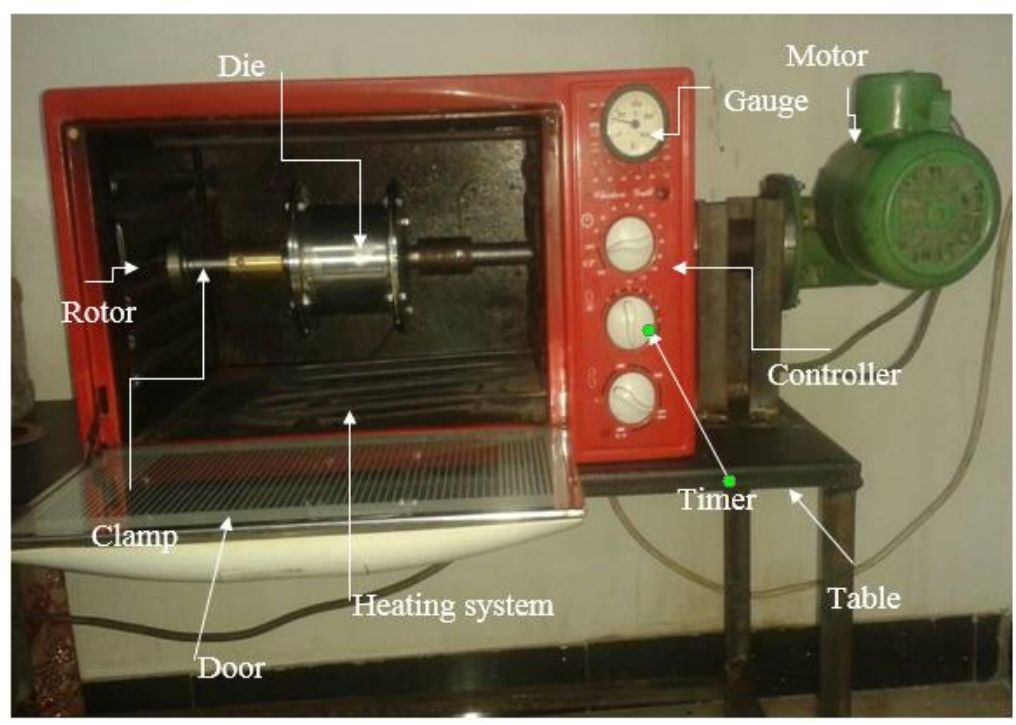

Figure 5: One-axis roto-mould system. 

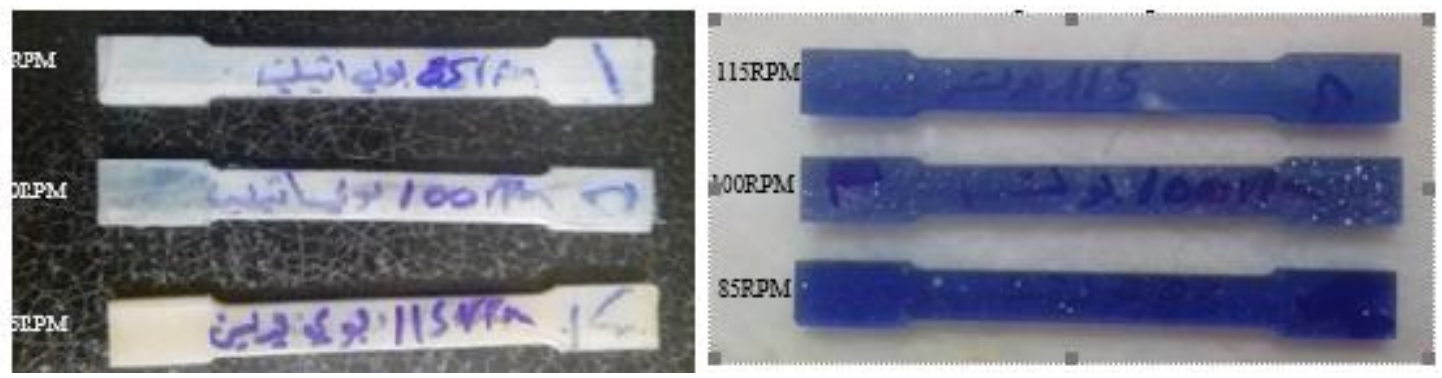

Figure 6: The specimens of tensile test
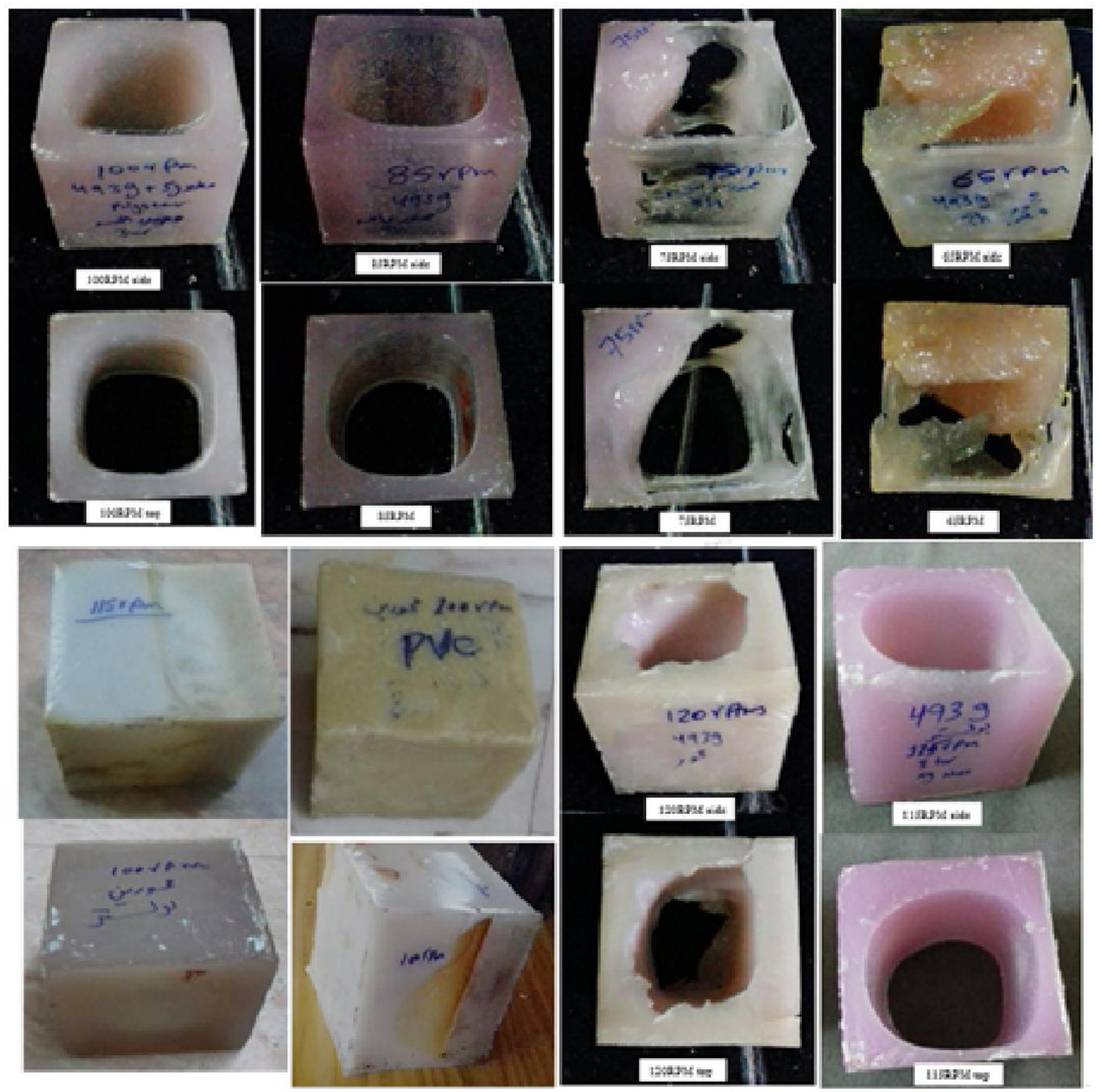

Figure 7: specimens with different RPM.

\section{EXPERIMENTAL RESULTS}

The study paying attention mainly to the rotational speed of the thickness and mechanical properties experimentally and theoretically and discuss the results. The results obtained from this study can be divided into three sections: at first, the results of work were done by used polyester for one axis with constant mass in different speeds at first limitation of this work is affect one axis of rotational speed to the shape. The second the results of work were done by used two axes (multi-axis) of rotational speed to achieve the effect of speed on the thickness, compressive ratio, tensile test, modulus of elasticity. Third; the results of work were done by used explosion method test for Polyethylene, PVC, polyester and polyethylene composite with different speed to achieve the effect of speed on the mechanical properties. Fourth; the results of work were done by using the production of the composite of polyethylene forced by iron roll wire, to achieve an improvement in that mechanical properties compared to the base material. The results obtained from this work as shown in figures 8-16. 


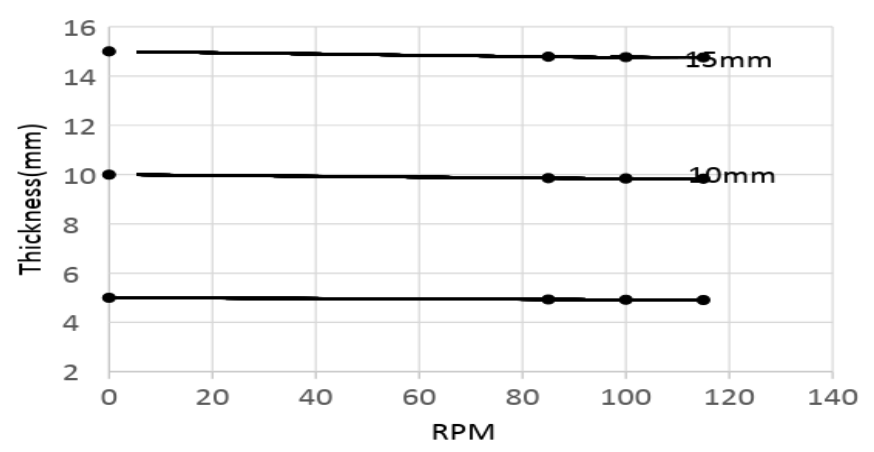

Figure (8) the change in average wall thickness of PVC.

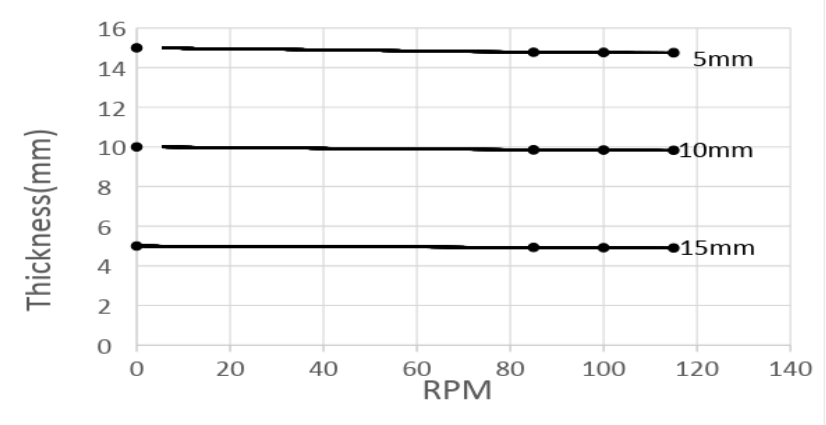

Figure (9) the change in average wall thickness of Polyethylene.

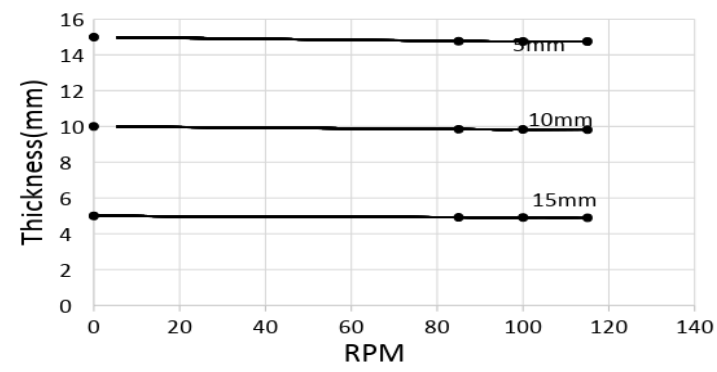

Figure (10) the change in average wall thickness of Polyester

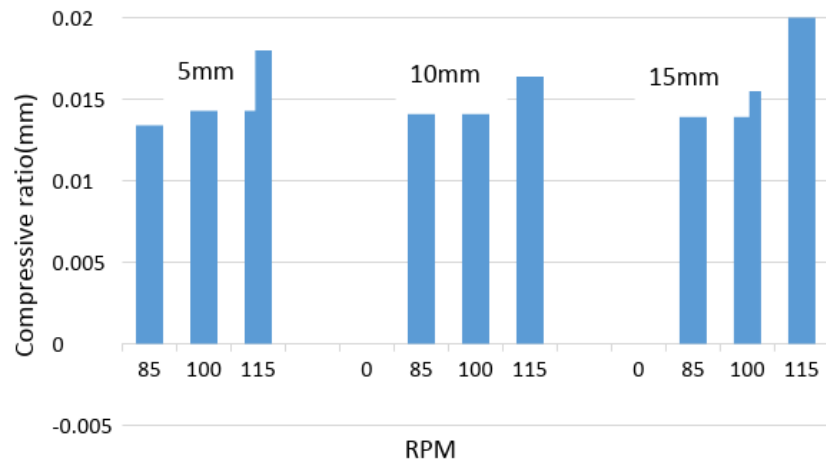

Figure 11: the compressive of PVC.

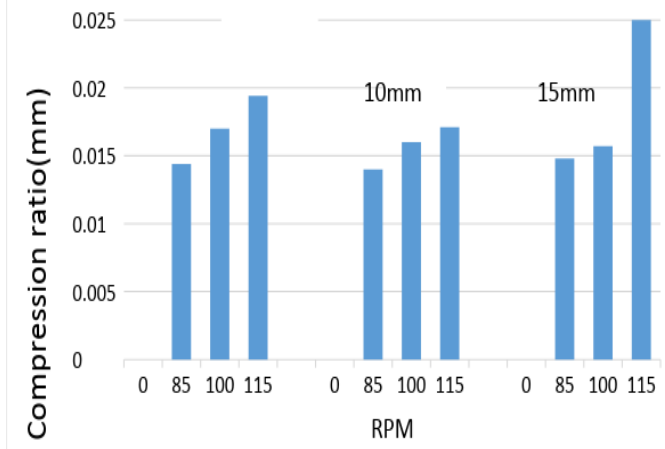

Figure 12: the compressive of Polyethylene

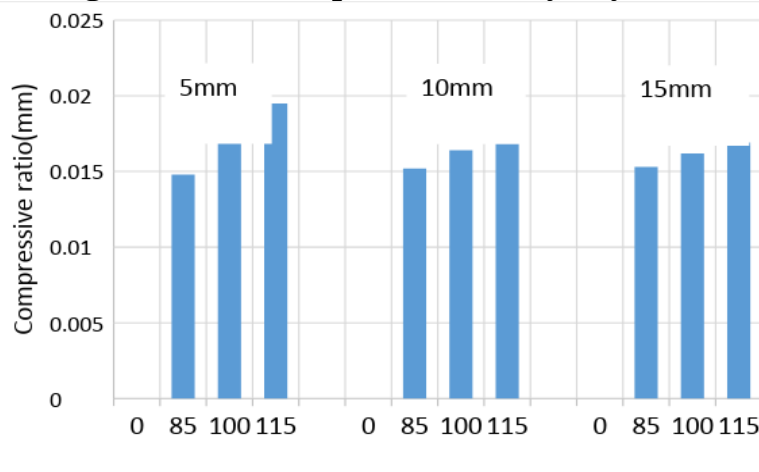

RPM

Figure 13: the compressive of Polyester.

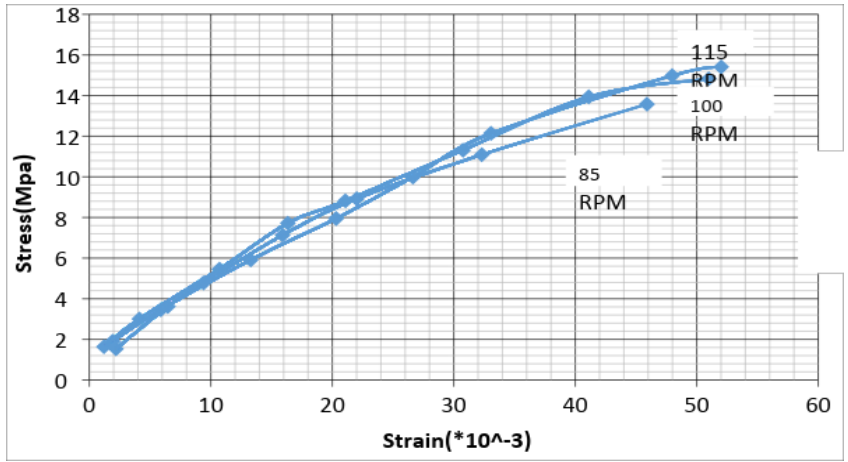

Figure 14: the tensile test of PVC. 


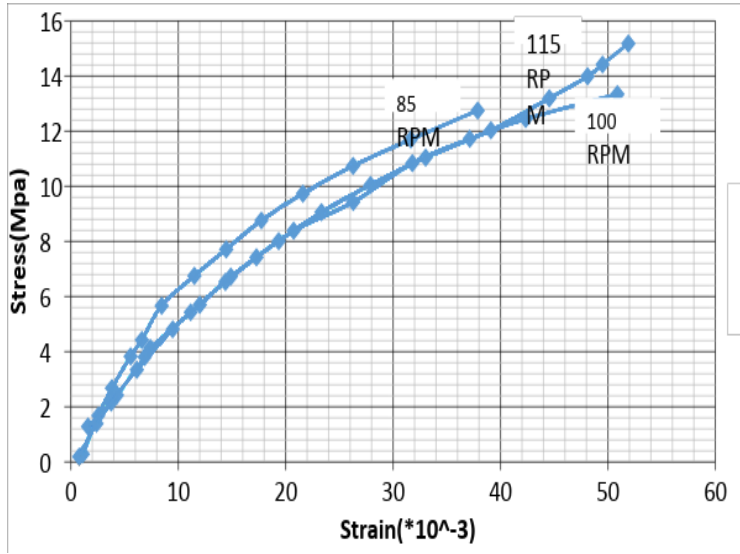

Figure 15: the tensile test of Polyethylene.

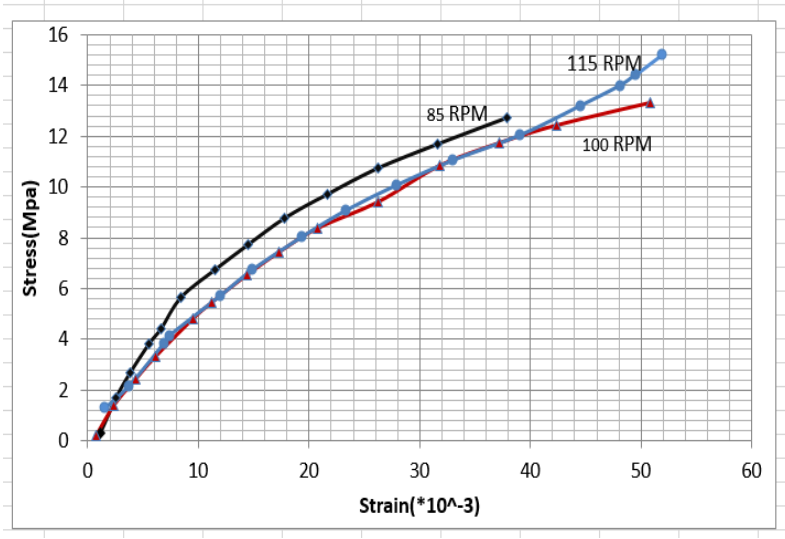

Figure 16: the tensile test roll of Polyethylene

\section{CONCLUSIONS}

This study is characterized by the following points: First: This study used multi-axis and different velocity (rotation of axis), while most researchers are satisfied with one axis or one speed. Second: As mentioned in the previous point, the study used multiple-axis with different velocity; thus, it was able to investigate their effects on the thickness of the wall. Third: In this study, closed cubic specimens were produced, and the explosion test was carried out in order to calculate the tensile strength for moulding at a variable speed.

\section{REFRENCES}

[1] Beal, G .1987 "Rotational Mould ing: Design "Material, Tolling and Processing" Munich, Germany, Hanser, Verlge.

[2] Dodge, P.T. and Perry, J.L. (2001) "Rotomould Part Density and its Relationship to Physical Properties" Proceeding ANTEC SPE 2001 plastic, the lone star volume 1: processing, may 6-10 2001 Dallas, Texas, society of plastics engineering.

[3] Crawford, R.J. and M.P. Kearns "Practical Guide to Rotational Mould ing" 2003: Rapra Technology Ltd.

[4] RAMKUMAR PL "Study on Mechanical and Fracture Behavior of Linear Low Density Polyethylene for Rotational Mould ing" Birla institute of technology and science, Pilani 2015.

[5] Crawford, R.J., et al., "Mould pressure control in rotational mould ing" Proceedings of the Institution of Mechanical Engineers, Part B: Journal of Engineering Manufacture, 2004. 218(12): p. 1683-1693.

[6] Yan, W., R.J.T. Lin, and D. Bhattacharyya "Particulate reinforced rotationally mould ed polyethylene composites Mixing methods and mechanical properties" Composites Science and Technology, 2006. 66(13): p. 2080-2088.

[7] Crawford, R.J. "Recent advances in the manufacture of plastic products by rotomould ing" Journal of Materials Processing Technology, 1996. 56(1-4): p. 263-271.

[8] R. J. Crawford, "Introduction to rotational moulding in Rotational moulding of plastics", Wiley, New York. 1992.

[9] Shih- Jung Liu and Ching- Hsiung Yang (2001): "Rotational moulding of two layered Polyethylene foams" Advances in Polymer Technology, Vol. 20, pp. 108-115.

[10] "Introduction to Rotational Mould ing" [Cited 26/5/07]; Available from: http://www.rotationalmoulding.com/Technical\%20Papers/Introduction\%2 0To\%20Rotational\%20Moulding.pdf.

[11] Martin, D., et al., "Polyethylene-layered silicate Nano composites for rotational mould ing" Polymer International, 2003. 52(11): p. 1774-1779.

[12] Carla F.M., Mohammad A.I and Vikas Prakash. (2007): "Dynamic fracture of linear Medium polyethylene under impact loading conditions" Materials Science and Engineering, Vol. 465, pp. 211-222. 
[13] Martin D et al "A summary of recent Australian R\&D activities in rotational mould ing" Rotation 2001(January-February):44-50.

[14] Crawford RJ, Robert A "Reinforcement of rotomould plastic parts - a challenge. The 3rd Asian-Australasian conference on composite materials (ACCM-3)" Auckland, New Zealand; 2002. p. 1 - 9.

[15] Harkin-Jones E, Crawford RJ "Mechanical properties of rotationally mould ed Nyrim" Polymer Engineering and Science 1996; 36(5):615-25.

[16] Yan W, Lin RJT, Bickerton S, Bhattacharyya D "Performance evaluation of mould ed polymeric composites" Polymer processing society Asia/Australia regional meeting (PPS-2002): G6, Thermoforming, Blow Moulding and Rotational Mould ing, Taipei, Taiwan; 2002, Paper No. 204.

[17] Yuze Han "Rotational moulding of a polyethylene resin: preliminary results on the effects of moulding conditions on the mould ed part characteristics" November, 2002.

[18] Laurenţiu Slătineanu1,*, Oana Dodun2, Margareta Coteaţă3, António ～Gonçalves-Coelho4, Irina Beşliu (Grigoraş) 5, Lorelei Gherman6, Maricela Poienaru7 "A Preliminary Study on the Rotational Moulding Process"Proceedings in manufacturing systems, volume 7, issue 1, 2012.

[19] Jachowicz1, Tomasz "Investigation Of The Influence Of Mould Rotational Speed On The Cast Wall Thickness In The Rotational Moulding Process" Science and Technology Research Journal. 2013, Vols. Volume 7, No. 19, pp. 79-87.

[20] CHAUDHARI, D M KULKARNI and V V "Parametric And Mechanical Characterization Of Linear Low. Indian Academy Of Sciences" Vol. 39, Part 3, June 2014, pp. 625-635.

[21] Dvorak, Maria"Applicability Of Recycled HDPE For Rotational Mould ing" Arcada . 2016, pp. 1-48.

[22] Drummer, Martin Löhner and Dietmar "Multi-Layer Rotational Moulding Of Pe-Pa Utilizing A Mulitphase Interlayer To Generate Mechanical Adhesion" SPE ANTECTM. 2016, pp. 1690- 1697.

[23] Roberto C. Vázquez Fletes, Pedro Ortega Gudiño,. "A Three-Layer Foamed Composite Prepared By Rotational Mould ing" SPE ANTECTM. 2016, pp. 1709- 1713.

[24] Rodrigue*, Alexandre Raymond and Denis. "Foams and Wood Composite Foams Produced By Rotomould ing. (C) Smithers Rapra Technology" 2013, Vols. Vol. 32, No. 4,

[25] Paul Betschart "Strengthening Rotationally Mould ed Products: Development of a Rotomouldable Thermoplastic Composite" March, 2008.

[26] F. J. Moscoso-Sánchez, J. Aguilar, R.M. Jiménez, J.R. Robledo-Ortíz, R. González-Núñez,. "Thermal Analysis Of The Rotational Moulding Cycle Followed By Internal Air Temperature Profiles: An Application For Foamed Polyethylene" SPE ANTECTM. 2016, pp. 1704-1708.

[27]Scribben, Eric "Selection Of Thermotropic Liquid Crystalline Polymers For Rotational Mould ing" Appendix F. Publications. 2014, pp. 1-382.

[28] E. ARCHER*, E. HARKIN-JONES AND M. P. KEARNS "The Rotational Moulding Characteristics of Metallocene Polyethylene Skin/Foam Structures" Journal of cellular plastics volume 43 — November 2007.

[29] Emad Samy Abdalla "Rapid Rotational Foam Moulding Of Integral Skin Polypropylene Cellular Composites" May 2009.

[30] Dixon Harold Abell "A Study of the Cause of Failure of Rotationally Mould ed, High-Density Polyethylene, Sodium Hypochlorite Storage Tanks" April 2011.

[31] Bei Wang "Pre-treatment of Flax Fibers for USE in .Rotationally Mould ed Biocomposite" July 2004. 
[32] E.O. Cisneros-López, M.E. González-López, A.A. Pérez-Fonseca, R. González-Núñez "Fiber Surface Treatment As An Approach To Increase Fiber Content In Agave-Lmdpe Composites Produced By Rotomould ing" SPE ANTEC ${ }^{\text {TM }}$. 2016, pp. $1681-1685$.

[33] N. Barhoumi1, A. Maazouz1*, M. Jaziri2, R. Abdelhedi2 "Polyamide from lactams by reactive rotational moulding via anionic ring-opening polymerization: Optimization of processing parameters" express polymer letters vol.7, no.1 (2013) 76-87.

[34] ORI1, Ottorino. "New Mould able And Water Soluble Cores For Hollow Composite Automotive Parts" Ottorino ORI1. 2016, pp. 1-6.

[35] J.M. Boyd, S. Wu, E.S. Takacs, M.R. Thompson, J. Vlachopoulos "Studying Polymer Particle Sintering With An Automated Imaging System" SPE ANTECTM. 2016.

[36] David Roylance "PRESSURE VESSELS "Cambridge, August 23, 2001.

[37] Serope Kalpakajian "Manufacturing engendering and technology processes" Hand Book Preface to Third Edition. Chicago, Illinois.

[38] Robert MJ "Mechanical of Composite Material" Hand Book Preface to Second Edition Printcd in the United States of America (1999). 